\title{
Experiences of Cancer-related Fatigue after Completion of Childhood Cancer Treatment: A Literature Review
}

Qamra Muaikel Alqahtani; King Saud University - College of Applied Medical Sciences, Rehabilitation- Physical Therapy, Saudi Arabia

\section{Introduction:}

Fatigue is the most frequent debilitating symptom that may persist for years in survivors of childhood cancer. After completion of cancer treatment, children reported fatigue as a barrier for engaging in physical activity and an obstacle to successful school re-entry.

\section{Objective:}

To explore the literature regarding the experience of cancer-related fatigue in survivors of childhood cancer who aged $\leq 18$ years at the time of participation in the research.

\section{Methods:}

Using the Joanna Briggs Institute guideline, an electronic search of the AMED, CINAHL, Cochrane, EMBASE, MEDLINE, and PsycINFO and reference lists was conducted. Data were extracted and assessed for quality.

\section{Results:}

*Four studies were included in this review with merely one study involved children younger than 13 years who survived brain tumours.

*After completion of cancer treatment, fatigue was perceived as a considerable problem (Edward et al. 2003 \& Ream et al. 2006) that caused activity intolerance and affected the ability to attend school (Macartney et al. 2014).

*Fatigue had an adverse impact on the ability to exercise (Spathis et al. 2017). * Most of children and adolescents managed fatigue by rest (Edward et al. 2003; Ream et al. 2006; Macartney et al. 2014), and exercise was the single best treatment for fatigue recommended by adolescents (Spathis et al. 2017).

*To the knowledge of the researcher, there is no study investigating parents' perspectives of how their children perceive and manage fatigue after completion of cancer treatment.

\section{Conclusions:}

Although fatigue is a distressing symptom reported by children who survived cancer, there has been limited research on fatigue experience among paediatric cancer survivors. Further research is needed to know how young children experience fatigue after completion of cancer treatment, and to explore parents' impact on fatigue management of their children. 